\title{
Advanced Linear Proportional Control of a Pressure Valve for an Electric Pressure Cooker
}

\author{
Kyung-Il Woo ${ }^{1}$ and Dae-Kyong $\mathrm{Kim}^{2}{ }^{2}$ \\ ${ }^{1}$ Pukyong National University, Busan, 608-737, Korea \\ ${ }^{2}$ Sunchon National University, Suncheon, 540-480, Korea \\ cogging@pknu.ac.kr,dkkim@sunchon.ac.kr
}

\begin{abstract}
This paper describes the advanced control of solenoid for pressure valve for electric pressure cooker. To remove the problem of the on/off non-proportional pressure control method which was used to control the existing solenoid, newly redesigned solenoid was adopted and the advanced control method, linear proportional control method, was proposed. The control system by the pressure sensor and AVR was manufactured and its validation was proved through the experiment.
\end{abstract}

Keywords: Electric cooker, linear proportional control, on/off non-continuous control, pressure valve, solenoid

\section{Introduction}

Domestic enterprises sell pressure control valves for electric cookers that are controlled by an on / off non-continuous method. On the other hand, the non-continuous pressure on / off control method of the high-performance electric pressure cooker that requires continuous and linear pressure control does not satisfy the pressure control performance. Few countries in Asia and Europe produce electric pressure cookers. The domestic technology level of electric pressure cookers is better than Japan technology level of the cookers. The general control valve technology of the United States and Europe is high, but there are no applications to the electric pressure cooker valves. Therefore, the development of the core technology rather than import the pressure control valve for the electric pressure cookers from foreign companies will improve the competitiveness of products. The rice cooker thermal control function is needed to satisfy precise pressure control but no pressure control valve has been developed to meet this performance.

This paper describes an advanced linear proportional control of the pressure valve by lowcost high-precision proportional control. The pressure valve is the moving coil type actuator. The response of the moving coil type actuator is faster because of the light weight [1-5]. The low cost of the AVR microcontrollers using the linear digital controller was designed to control the pressure control solenoid, and the superiority of the proposed controller was confirmed experimentally.

* Corresponding Author; Tel: +82-61-750-3546, Fax:+82-61-753-3546, E-mail:dkkim@ sunchon.ac.kr 


\section{Solenoid for Pressure Control Valve}

\subsection{Solenoid}

The thrust generated by the solenoid can be expressed in equation (1), which was obtained from the magnetic flux generated by the permanent magnet and the current.

$$
F=N B_{g} l \times i[N]
$$

The magnetic flux density in the air gap, $B_{g}$, is the sum of those by the magnet, $B_{m}$, and the coil, $B_{\text {coil }}$. Therefore, the thrust of the solenoid can be calculated using equation (2)

$$
F=\operatorname{Nli}\left(B_{m}+B_{g}\right)[N] .
$$

The magnetic flux density generated by the coil is

$$
B_{\text {coil }}=\frac{N i \mu_{0}}{2\left(l_{m}+l_{g}\right)}\left[W b / m^{2}\right] .
$$

Equation (4) can be obtained using equations (2) and (3). Figure 1 shows the non-linear characteristics of the solenoid. In this figure, the linear and nonlinear characteristics were indicated by the permanent magnet and coil, respectively.

$$
F=N l i B_{m}+N l\left[\frac{\mu_{0}}{2\left(l_{m}+l_{g}\right)}\right] i^{2}[N]
$$

Figure 1 shows that in the solenoid, the magnetic flux direction by the coil and the direction of the magnetic flux generated by the permanent magnet are different, which causes a pushing and pulling force.

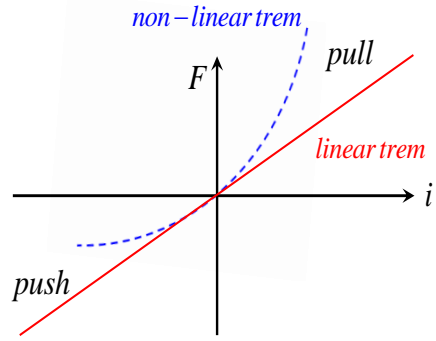

Figure 1. Nonlinear characteristics of the solenoid

\subsection{Motion Equation of Solenoid}

When the mover of the solenoid is moved by the thrust generated in the direction, as shown in Figure 2, the voltage equation can be expressed as equation (5).

$$
V_{0}=R i+\frac{d \lambda}{d t}=R i+L \frac{d i}{d t}+k_{c} x^{\prime}[V]
$$




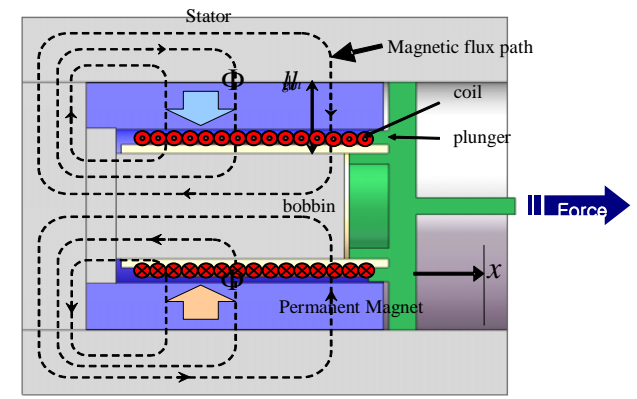

\section{Figure 2. Thrust generation of the solenoid}

The motion equation of the mover is expressed in equation (6).

$$
F=m \frac{d x^{\prime}}{d t}+k_{d} x^{\prime}+F_{f}+k_{s} x+F_{\text {const }}[N]
$$

where,

$m$ : weight of the mover,

$k_{d}$ : damping coefficient by air,

$F_{f}:$ resistance coefficient by the plunger,

$k_{s}$ : spring coefficient by the spring,

$F_{\text {const }}:$ constant force by gravity,

$x^{\prime}: \frac{d x}{d t}$.

Equation (7) can be derived by summation of the thrust by the current and equation (6).

$$
\begin{gathered}
m \frac{d x^{\prime}}{d t}=-\left(F_{f}+F_{\text {const }}\right)-\left(k_{d} x^{\prime}+k_{s} x-N B_{g} l i\right) . \\
L \frac{d i}{d t}=V_{0}-\left(k_{e} x^{\prime}+R i\right) .
\end{gathered}
$$

The position of the solenoid can be calculated using these equations according to the voltage control method. In this equation, $V_{0}$ is the DC link voltage.

\section{Implementation of the Digital Controller}

A high precision digital controller for the linear proportional valve was implemented using a pressure sensor. The digital controller was realized by controlling the valve current because the linear proportional valve has linear thrust characteristics for the input current. The digital controller was simplified considering the low- cost product.

Figure 3 shows the structure of the digital controller for the pressure cooker. The low-cost pressure sensor, BOSCH BMD070, was used to measure the pressure of the cooker, and the measured analog pressure signal was changed to the digital data by the low-cost microcontroller AVR128 data acquisition system. Finally, the closed-loop pressure controller was implemented to control the position and thrust of the linear proportional control valve by controlling the current flowing into the coil of the valve mover and digital PI controller. Figure 4 shows the circuit of the developed digital controller. Figure 5 and 6 show the Antialias filter circuit for ADC and frequency characteristic of Anti-alias filter, respectively. Figure 7 presents the prototype of the controller according to pressure and current control mode. Figure 8 shows the position control test of the pendulum of the cooker according to the 
current. Figure 9 shows the experimental set-up for pressure control using the developed controller and linear proportional valve, as well as the commercial pressure cooker. The existing on/off pressure control method and proposed linear proportional control method were used to control the internal pressure of the cooker (0.7[Bar]). In addition, to measure the internal pressure of the cooker, a separate pressure sensor was attached to the outside of the cooker, and the control precision was confirmed using an oscilloscope.

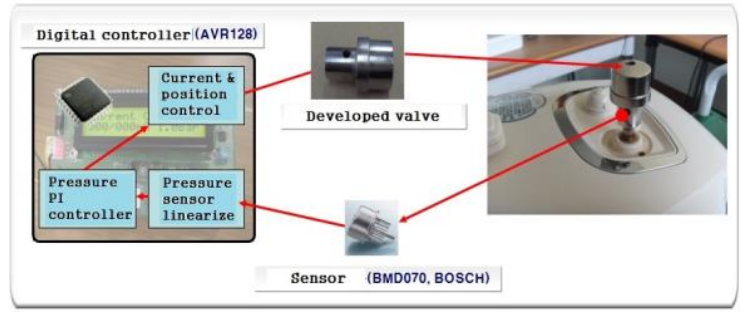

\section{Figure 3. Configuration of the digital controller}

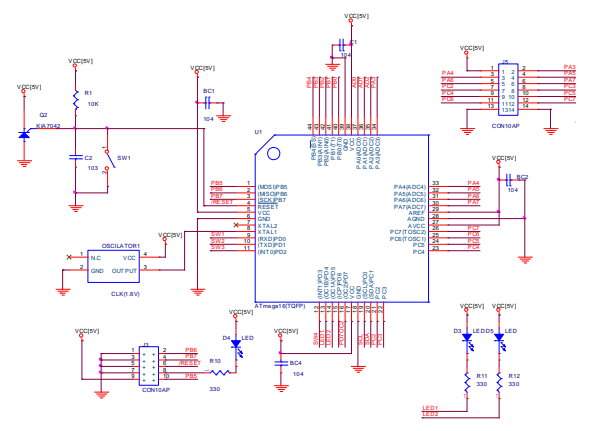

(a) Circuit of the micro-controller part

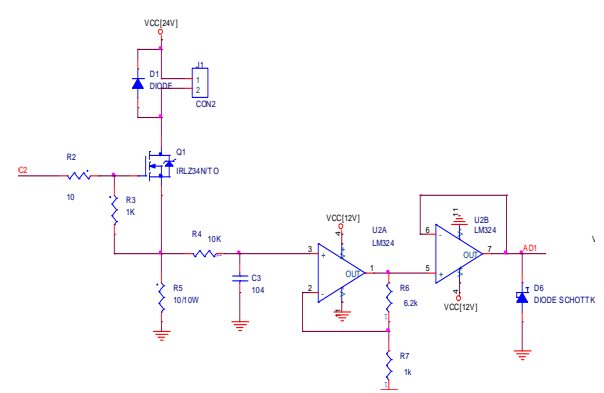

(b) Circuit of the output current part

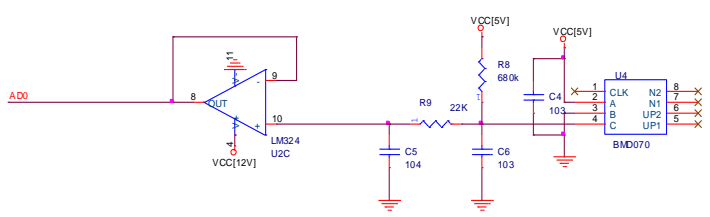

(c) Circuit of the pressure sensor measurement part 


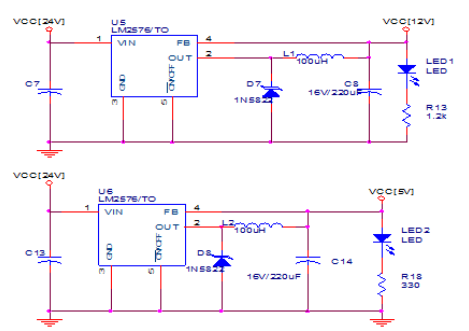

(d) Circuit of power supply part

Figure 4. Circuit of the digital controller

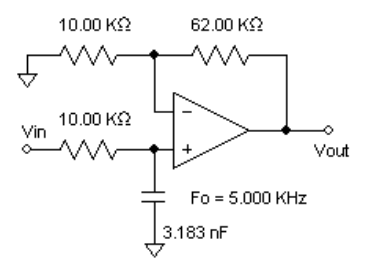

Figure 5. Anti-alias filter circuit for ADC

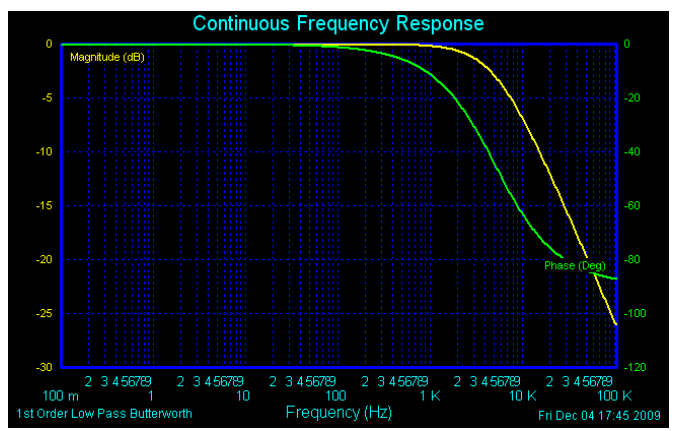

Figure 6. Frequency characteristic of Anti-alias filter

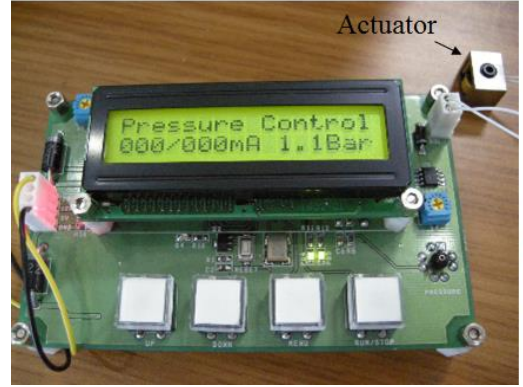

(a) Pressure control mode

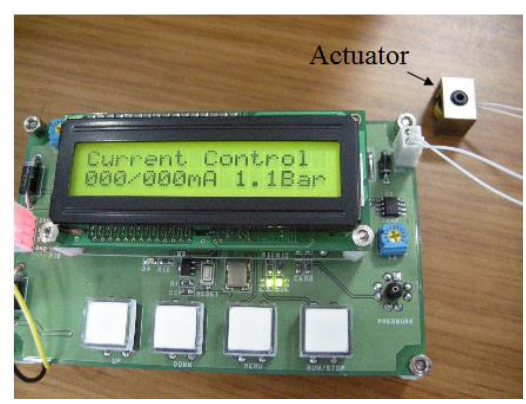

(b) Current control mode

Figure 7. Prototype of the controller according to control mode 


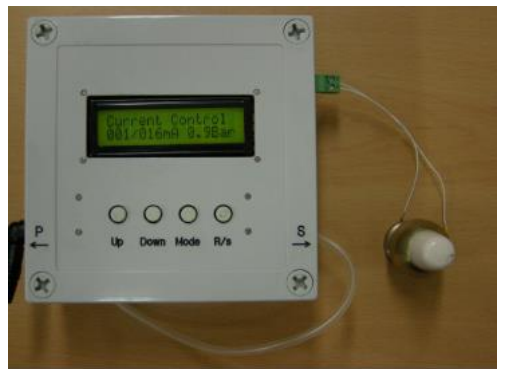

(a) Assembled valve and pendulum

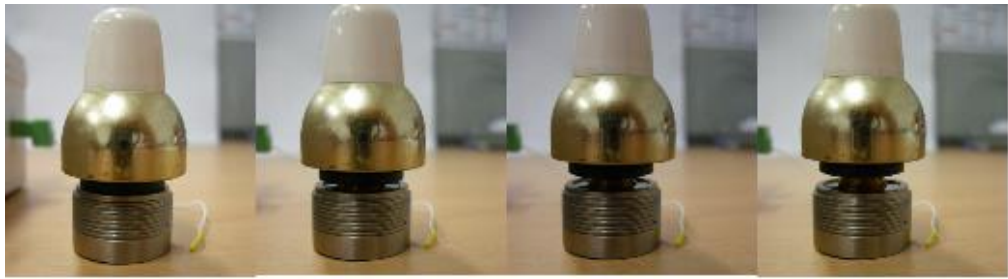

(b) Movement of the pendulum according to the current

Figure 8. Position control test according to the current

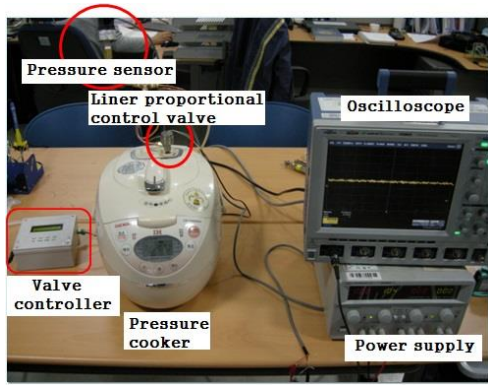

Figure 9. Experimental set for pressure control

Figure 10 compares the pressure control according to the pressure control method. Figure 10 (a) presents the results of the pressure control by the existing on / off control method; the maximum pressure variation is $0.1[\mathrm{Bar}$ ], and the minimum degree of deviation is 0.05 [Bar]. On the other hand, in figure 10 (b), the maximum pressure variation is 0.085 [Bar], and the minimum degree of deviation is $0.03[\mathrm{Bar}$. These results show that the proposed control method allows better control of the internal pressure of the cooker.
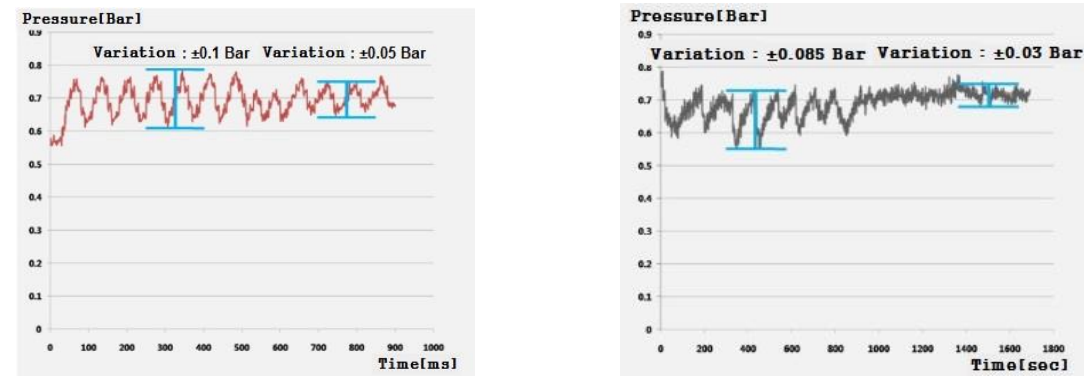

(a) Pressure control of the existing method) (b) Pressure control of the developed valve(on/off pressure control valve (linear pressure control method)

Figure 10. Pressure control comparison according to the pressure control method 


\section{Conclusion}

This paper described a system for advanced proportional control of the pressure valve in an electric pressure cooker. To overcome the problem of the existing on/off non-proportional pressure control method, a new high precision linear proportional valve digital controller was implemented using a low-cost pressure sensor and an AVR microcontroller. The experimental results validated the proposed method, and the pressure variation of the pressure using the proposed method was reduced.

\section{Acknowledgements}

This study was supported by a Research Grant of Pukyong National University (2013Year)

\section{References}

[1] Boldea and S. A. Nasar, "Linear Electric Actuators and Generators", Cambridge University Press, (1997).

[2] S. S. Jeong and S. M. Jang, "Armature reaction effect and inductance of moving coil linear oscillatory actuator with unbalanced magnetic circuit", IEEE trans. Magn., vol. 37, Issue 4, Part 1, (2001) July.

[3] S. M. Jang, "Static Characteristics of a Moving-Coil-Type Linear Motor in Consideration of Interaction between PM and Armature Field", KIEE International Transactions on Electrical Machinery and Energy Conversion System, vol. 48B, no. 1, (1999), pp. 19 29.

[4] G. H. Kang, J. P. Hong, G. T. Kim and D. H. Kim, "An Approach Toward Improving Performance of Moving Coil Type Linear Oscillatory Actuator Considering Asymmetric Magnetic Circuit”, Industry Applications Conference, vol. 1, (2000).

[5] E. A. Mendrela and Z. J. Pudlowski, "Transients and dynamics in a linear reluctance self-oscillating motor", IEEE Trans. Energy Conversion, vol. 7, no. 1, (1992).

[6] Canova, M. Ottella and D. Rodger, "A coupled field-circuit approach to 3D FEM analysis of electromechanical devices", Electrical Machines and Drives on Ninth International Conference, (1999), pp. 71-75. 
International Journal of Control and Automation Vol.7, No.3 (2014) 